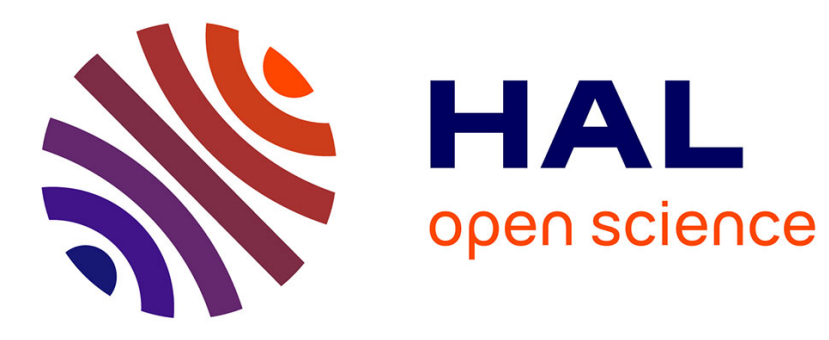

\title{
Marche aléatoire sur un di-graph et frontière de Martin
}

Basile de Loynes

\section{To cite this version:}

Basile de Loynes. Marche aléatoire sur un di-graph et frontière de Martin. Comptes rendus de l'Académie des sciences. Série I, Mathématique, 2012, 350 (1-2), pp.87-90. 10.1016/j.crma.2011.12.005 . hal-00677764

\section{HAL Id: hal-00677764 https://hal.science/hal-00677764}

Submitted on 24 Mar 2012

HAL is a multi-disciplinary open access archive for the deposit and dissemination of scientific research documents, whether they are published or not. The documents may come from teaching and research institutions in France or abroad, or from public or private research centers.
L'archive ouverte pluridisciplinaire HAL, est destinée au dépôt et à la diffusion de documents scientifiques de niveau recherche, publiés ou non, émanant des établissements d'enseignement et de recherche français ou étrangers, des laboratoires publics ou privés. 


\title{
Random walks on oriented lattices and Martin boundary
}

\author{
B. de Loynes
}

\section{Abridged English version}

In this note, we aim at sudying the Martin boundary of a simple random walk on a directed lattice. More precisely, let us denote by $\left(e_{1}, e_{2}\right)$ the canonical basis of $\mathbb{Z}^{2}$, and by sgn the sign function on the integers which is equal to -1 on negative integers and 1 elsewhere. Thus, we are considering a $\mathbb{Z}^{2}$-valued Markov chain $\left(M_{n}\right)_{n \geq 0}$ with Markov operator $P$ defined for $(u, v) \in \mathbb{Z}^{2} \times \mathbb{Z}^{2}$ as follows

1. $P(u, v)=\frac{1}{3}$ if $u_{2} \neq 0$ and either $v=u \pm e_{2}$ or $v=u+\operatorname{sgn}\left(u_{2}\right) e_{1}$;

2. $P(u, v)=\frac{1}{2}$ if $v=u \pm e_{2}$ and $u_{2}=0$.

For this random walk, the following can be shown.

Theorem 2.1 The Martin boundary of the simple random walk $\left(M_{n}\right)_{n \geq 0}$ is trivial : all positive harmonic functions are constant.

In our case, the triviality of the Martin boundary comes from precise estimates of the Green function. Two kinds of behavior can be observed ; let $z \in \mathbb{Z} \times\{0\}$, then there exist positive constants $c$ and $s(\lambda)$ such that

1. $G(z, y) \sim s(\lambda)\left|y_{2}\right|^{-1}$ if $y_{1} y_{2}^{-2}$ converges to $\lambda \in \mathbb{R}$

2. $G(z, y) \sim c\left|y_{1}-z\right|^{-\frac{1}{2}}$ if $y_{1} y_{2}^{-2}$ goes to $\pm \infty$.

The fact that we give estimates of the Green function only for $z \in \mathbb{Z} \times\{0\}$ comes from technical difficulties which can be removed by the formula (2) of section 4 .

The triviality of the Martin boundary implies the triviality of the Poisson boundary, thus all bounded harmonic functions are constant (see [4] and references therein for a modern approach). Actually, ideas in [2] or in [7] can be suited to the Markov operator we are considering in this note and allows a direct proof of the triviality of the Poisson boundary. We do not follow this approach here because we obtain the stronger result of triviality of the Martin boundary.

\section{Introduction}

Un di-graphe $\mathbf{G}$ est la donnée d'un couple $(\mathbf{V}, \mathbf{E})$ d'un ensemble dénombrable $\mathbf{V}$ de points et d'un sous-ensemble $\mathbf{E} \subset \mathbf{V} \times \mathbf{V}$ d'arêtes orientées. Si $e \in \mathbf{E}$ est une arête, on note $s(e)$ (resp. $t(e))$ le point source (resp. le point terminal). Plus précisément, $s(e)=u$ et $t(e)=v$ si $e=(u, v)$. Pour chaque point $u \in \mathbf{V}$, on définit le degré sortant de $u$, et on le note $d_{u}^{-}$le nombre d'éléments de l'ensemble $\{e \in \mathbf{E}: s(e)=u\}$. Un di-graphe est dit transitif si pour tout point $u, v \in \mathbf{V}$ il existe une suite finie de points $\left\{w_{0}, \cdots, w_{k}\right\}$ vérifiant $w_{0}=u, w_{k}=v$ et $\left(w_{i}, w_{i+1}\right) \in E$ pour tout $i \in\{0, \cdots, k-1\}$.

Définition 1.1. Soit $\mathbf{G}=(\mathbf{V}, \mathbf{E})$ un di-graphe. La marche aléatoire simple sur $\mathbf{G}$ est la chaîne de Markov à valeurs dans $\mathbf{V}$, notée $\left(\left(M_{n}\right)_{n>0}, P, \mu\right)$ où $\mu$ est une probabilité sur $\mathbf{V}$, et $P$ le noyau de Markov défini par $P(u, v)=\frac{1}{d_{u}^{-}}$si $(u, v) \in \mathbf{E}$ et 0 sinon. 
Dans cette note, on considère les treillis bi-dimensionnels, i.e. on supposera que $\mathbf{V}=\mathbb{Z}^{2}$ et que $\mathbf{E}$ est un sous-ensemble de l'ensemble des proches voisins dans $\mathbb{Z}^{2}$. On décompose $\mathbf{V}=\mathbf{V}_{1} \times \mathbf{V}_{2}$ en les directions horizontales et verticales, c'est à dire, si $v \in \mathbf{V}$, on écrit $v=\left(v_{1}, v_{2}\right)$ avec $v_{1} \in \mathbf{V}_{1}$ et $v_{2} \in \mathbf{V}_{2}$.

Définition 1.2. Soient $\mathbf{V}=\mathbf{V}_{1} \times \mathbf{V}_{2}$ et $\left(\epsilon_{y}\right)_{y \in \mathbf{V}_{2}}$ à valeurs dans $\{-1,0,1\}$. On appelle treillis $\epsilon$-orienté, et on le note $\mathbf{G}=(\mathbf{G}, \epsilon)$, le graphe orienté dont l'ensemble $\mathbf{V}$ de points est donné par $\mathbf{Z}^{2}$ et $(u, v) \in \mathbf{E}$ est une arête orientée si et seulement si l'une des conditions suivantes est vérifiées :

- soit $v_{1}=u_{1}$ et $v_{2}=u_{2} \pm 1$;

- soit $v_{2}=u_{2}$ et $v_{1}=u_{1}+\epsilon_{u_{2}}$ si $\epsilon_{u_{2}} \neq 0$.

Dans cette note, on étudiera le graphe $\mathbb{H}=(\mathbf{G}, \epsilon)$ où l'orientation $\epsilon$ est définie par $\epsilon_{0}=0$ et $\epsilon_{y}=\operatorname{sgn}(y)$ où sgn est la fonction signe. Il est clair que $\mathbb{H}$ est transitif, ainsi la marche aléatoire simple sur $\mathbb{H}$ est irréductible.

Les marches aléatoires sur ce type de graphe sont étroitement liées aux diffusions en milieu poreux. On pourra lire avec intérêt [5] où ce point de vue est développé.

\section{Résultat principal}

Soit $\left(M_{n}\right)_{n \geq 0}$ la marche aléatoire simple sur $\mathbb{H}$. On notera $\mathbf{P}^{\mu}$ la probabilité, sur l'espace des trajectoires, associée au noyau $P$ et plus simplement $\mathbf{P}^{x}$ si $\mu$ est la masse de Dirac en $x \in \mathbf{V}^{2}$. On définit par récurrence la suite de temps d'arrêt $\left(\tau_{n}\right)_{n \geq 0}$ par $\tau_{0}=0$ et $\tau_{n+1}=\inf \left\{t \geq \tau_{n}+1\right.$ : $\left.M_{t}^{(2)}=0\right\}$ où $M_{t}=\left(M_{t}^{(1)}, M_{t}^{(2)}\right)$. Il est facile de voir que $\tau_{n}<\infty \mathbf{P}^{x}$-p.s. pour tout $n \geq 0$. La suite de variables aléatoires $\left(M_{\tau_{n}}\right)_{n \geq 0}$ est alors elle-même une chaîne de Markov que l'on appellera chaîne de Markov induite. Par définition, $M_{\tau_{n}}^{(2)}=0$, ainsi, si $\mu$ est à support dans $\mathbf{V}_{1} \times\{0\}$, noté par la suite $\mathcal{V}_{0}$, la chaîne de Markov induite est confinée dans ce sous-ensemble $\mathcal{V}_{0}$. La chaîne de Markov induite sera alors, selon le contexte, considérée comme une chaîne de Markov à valeurs dans $\mathbb{Z}^{2}$ ou $\mathbb{Z}$.

Dans [1], il est montré que la chaîne de Markov originale est transiente, et cela est une conséquence de la transience de la chaîne de Markov induite qui peut-être vue comme une marche aléatoire sur $\mathbb{Z}$ à sauts non bornés. Ainsi, l'étude de la frontière de Martin de ces chaînes de Markov est pertinente.

Théorème 2.1. Les frontières de Martin des chaînes de Markov induites et originales sont triviales, i.e. les seules fonctions harmoniques positives sont les fonctions constantes.

\section{Frontière de Martin de la chaîne de Markov induite}

La compactification de Martin est longuement présentée dans [8]. Essentiellement, la compactification de Martin fait intervenir le noyau de Martin : si o est un point-base du graphe $\mathbb{H}$, le noyau de Martin est défini pour $x, y \in \mathbb{H}$ par $K(x, y)=\frac{G(x, y)}{G(o, y)}$ où $G$ est la fonction de Green associée à la chaîne de Markov. Ainsi, des estimées précises de $G$ permettent de donner une description de la frontière de Martin.

Définition 3.1. Soient $(\psi)_{n \geq 0}$ une suite de variables aléatoires indépendantes et identiquement distribuées à valeurs dans $\{-1,1\}$ de loi de Bernouilli de paramètre $\frac{1}{2}$ et $\left(Y_{n}\right)_{n \geq 0}$ une suite de variables aléatoires définies par $Y_{0}=M_{0}^{(2)}$ et $Y_{n}=Y_{0}+\sum_{k=0}^{n-1} \psi_{k}$. On note $\eta_{n}(y)$ le temps local de $\left(Y_{n}\right)_{n \geq 0}$ en $y \in \mathbb{Z}$, i.e. $\eta_{n}(y)=\sum_{k=0}^{n} 1_{Y_{k}=y}$.

Définition 3.2. Soit $\left(\sigma_{n}\right)_{n \geq 0}$ une suite de temps d'arrêt définie par $\sigma_{0}=0$ et $\sigma_{n+1}=\inf \{t \geq$ $\left.\sigma_{n}+1: Y_{t}=0\right\}$. 
Définition 3.3. Soit $\left(\xi_{n}^{(y)}\right)_{n \geq 1, y \in \mathbf{V}_{2}}$ une suite de variables aléatoires indépendantes et identiquement distribuées à valeurs dans $\mathbb{N}$ de loi géométrique de paramètre $p=\frac{1}{3}=1-q$ et pour $n \geq 0$ posons $X_{n}=\sum_{y \in \mathbf{V}_{2}} \epsilon_{y} \sum_{i=1}^{\eta_{\sigma_{1}-1}(y)} \xi_{i}^{(y)}$. Enfin, on note par $T_{n}$ le temps défini par $T_{n}=$ $n+\sum_{y \in \mathbf{V}_{2}} \sum_{i=1}^{\eta_{\sigma_{1}-1}(y)} \xi_{i}^{(y)}$, avec la convention usuelle $\sum_{\emptyset}=0$.

Dans [1], il est montré que $M_{T_{n}} \stackrel{\text { loi }}{=}\left(X_{n}, Y_{n}\right)$ et $\tau_{n} \stackrel{\text { loi }}{=} T_{\sigma_{n}}$. Ceci permet de calculer la loi de $M_{\tau_{n}}$.

Proposition 3.4. La loi de $M_{\tau_{n}}$ est déterminée par sa fonction caractéristique $\mathbf{E}^{0}\left(e^{i\left\langle t, M_{\tau_{n}}\right\rangle}\right)=$ $\mathbf{E}^{0}\left(e^{i t_{1} X_{\sigma_{1}}}\right)^{n}$. De plus, la fonction caractéristique de $X_{\sigma_{1}}$ est donnée par $\phi(t)=\operatorname{Re} r(t)^{-1} g(r(t))$ où $r(t)=\left[3-2 e^{i t}\right]^{-1}$ et $g(x)=\frac{1-\sqrt{1-x^{2}}}{x}$.

En particulier, la fonction de Green associée à la chaîne de Markov $\left(M_{\tau_{n}}\right)_{n \geq 0}$ est donnée, pour $u, v \in \mathcal{V}_{0}$, par $G_{0}(u, v)=G_{0}(0, v-u)=(2 \pi)^{-1} \int_{-\pi}^{\pi} e^{-i t(v-u)}[1-\phi(t)]^{-1} d t$. La fonction $[1-\phi(\cdot)]^{-1}$ possède, en 0 , une singularité en $\frac{1}{\sqrt{|t|}}$. Une décomposition en séries permet de traiter cette singularité à part et de trouver l'équivalent suivant.

Proposition 3.5. Il existe une constante $c>0$ telle que $G_{0}(u, v)=G_{0}(0, v-u) \sim c|v-u|^{-\frac{1}{2}}$ lorsque $|v|$ tend vers l'infini.

Ainsi le noyau de Martin $K_{0}(u, v)=\frac{G_{0}(u, v)}{G_{0}(0, v)}$ possède une unique valeur d'adhérence lorsque $|v|$ tend vers l'infini ce qui implique la trivialité de la frontière de Martin.

\section{Frontière de Martin de la chaîne de Markov originale}

Dans ce paragraphe on s'intéresse à la frontière de Martin de la chaîne originale. On notera $G$ (resp. $K$ ) la fonction de Green (resp. le noyau de Martin) associée à la marche originale.

On note $\eta_{s, t}(y)$, pour $s, t \geq 0$ et $y \in \mathbb{H}$, le temps local de $\left(M_{n}\right)_{n \geq 0}$ en $y$, i.e. $\eta_{s, t}(y)=$ $\sum_{k=s}^{t-1} 1_{M_{n}=y}$ toujours avec la convention $\sum_{\emptyset}=0$. On peut alors exprimer le noyau de Martin de la chaîne originale en fonction de celui de la chaîne induite.

$$
K(x, y)=\left\{\begin{array}{l}
K_{0}(x, y) \text { si }(x, y) \in \mathcal{V}_{0} \\
\frac{\mathbf{E}^{x}\left(\eta_{0}, \tau_{1}(y)\right)}{G(0, y)}+\sum_{z \in \mathbb{Z}} \nu_{x}(z) K(z, y) \text { sinon }
\end{array}\right.
$$

où $\nu_{x}(z)=\mathbf{P}^{x}\left(M_{\tau_{1}}=(z, 0)\right)=\mathbf{P}^{x}\left(X_{\sigma_{1}}=z\right)$.

En utilisant les symétries de $\mathbb{H}$, en particulier que ce graphe est invariant par les translations horizontales et par les symétries centrales de centre situé sur $\mathcal{V}_{0}$, on peut montrer la proposition suivante.

Proposition 4.1. Soient $z \in \mathcal{V}_{0}$ et $y \in \mathbb{H}$, alors la fonction de Green $G$ est donnée par

$$
G(z, y)=(2 \pi)^{-1} \int_{-\pi}^{\pi} e^{i t\left(y_{1}-z_{1}\right)} \frac{g(r(t))^{\left|y_{2}\right|}}{1-\phi(t)} d t
$$

où $r, g$ et $\phi$ sont définies dans le paragraphe 3.

Il est alors possible d'obtenir les estimées suivantes de la fonction de Green.

Proposition 4.2. Soient $z \in \mathcal{V}_{0}, y \in \mathbb{H}$ et supposons que $y_{1} y_{2}^{-2}$ converge vers $\lambda \in \mathbb{R} \cup\{ \pm \infty\}$, alors il existe pour tout $\lambda$ une constante $s(\lambda)>0$ telle que

1. $G(z, y) \sim s( \pm \infty)\left|y_{1}-z_{1}\right|^{-\frac{1}{2}}$ lorsque $y_{1} y_{2}^{-2}$ tend vers $\pm \infty ;$

2. $G(z, y) \sim s(\lambda)\left|y_{2}\right|^{-1}$ lorsque $y_{1} y_{2}^{-2}$ converge vers $\lambda$.

Par un calcul direct, on peut montrer qu'il est possible de passer à la limite dans la somme $\sum_{z \in \mathbb{Z}} \nu_{x}(z) K(z, y)$, d'où la proposition suivante. 
Proposition 4.3. Soit $x \in \mathbb{H}$, alors $\sum_{z \in \mathbb{Z}} \nu_{x}(z) K(z, y)$ possède une unique valeur d'adhérence lorsque $|y|$ tend vers l'infini.

Proposition 4.4. La quantité $\mathbf{E}^{0}\left(\eta_{0, \tau_{1}}(y)\right)$ décrồt vers 0 en o $\left(\left|y_{1}\right|^{-\frac{1}{2}}\right)$ lorsque $y_{1} y_{2}^{-2}$ tend vers $\pm \infty$ et en $o\left(\left|y_{2}\right|^{-1}\right)$ lorsque $y_{1} y_{2}^{-2}$ converge.

Par les propositions 4.2 et 4.4 , le premier terme dans l'équation (2) converge vers 0 lorsque $|y|$ tend vers l'infini ; et par la proposition 4.3, le deuxième terme de l'équation (2) converge vers 1 lorsque $|y|$ tend vers l'infini. Enfin, le terme de l'équation (1) tend vers 1 lorsque $|y|$ tend vers l'infini par la proposition 3.5. Ainsi, le noyau de Martin $K$ possède une unique valeur d'adhérence à l'infini ce qui montre la trivialité de la frontière de Martin de la chaîne originale.

Dans le cas où $\epsilon$ est une suite de variables aléatoires indépendantes et identiquement distribuées, il est montré dans [1], que la marche aléatoire simple est encore transiente (pour presque toute orientation). Ce résultat a été généralisé dans [3] où est considéré le cas où $\left(f_{y}\right)$ est une suite stationnaire à valeurs dans $[0,1]$ et où $\epsilon_{y}$ est pris égal à 1 avec probabilité $f_{y}$ et -1 avec probabilité $1-f_{y}$. Sous la condition $\mathbf{E}\left(f_{0}\left(1-f_{0}\right)\right)^{-1 / 2}<\infty$, la marche simple est transiente. Enfin, dans [6], est considéré le cas où l'orientation $\epsilon$ est une suite stationnaire vérifiant certaines conditions de décorrélations. En moyenne, une orientation aléatoire est moins dégénérée que l'orientation choisie dans cette note, on peut donc s'attendre, dans ce cadre, à la trivialité des frontières de Martin pour presque toute orientation.

\section{References}

[1] M. Campanino, D. Petritis, Random walks on randomly oriented lattices, Markov Process. Related Fields, 9, 2003, 394-412.

[2] G. Choquet, J. Deny, Sur l'équation de convolution $\mu=\mu \star \sigma$, C.R. Acad. Sci. Paris, 250, 1960, 799-801.

[3] N. Guillotin-Plantard, A. Le Ny, Transient random walks on 2D-oriented lattices, Teor. Veroyatn. Primen., 52, 2007, 815-826.

[4] V. Kaimanovich, Measure-theoretic boundaries of Markov chains, 0-2 laws and entropy, Proceedings of the Conference on Harmonic Analysis and Discrete Potential Theory (M.A. Picardello, ed.), 1992, 145-180.

[5] G.Matheron, G. de Marsily, Is transport in porous media always diffusive ? a counter-example, Water Resour. Res., 16, 1980, 901-917.

[6] F. Pène, Transient random walk in $\mathbb{Z}^{2}$ with stationary orientations, ESAIM Probab. Stat., 13, 2009, 417-436.

[7] F. Spitzer, Principles of Random walk, 2nd edition, Springer-Verlag, 2001.

[8] W. Woess, Denumerable Markov chains, EMS Textbooks in Mathematics, 2009. 